\title{
Comparing Two Treatment Approaches for Patients with Type 1 Diabetes During Aerobic Exercise: A Randomised, Crossover Study
}

\section{Varun Vartak}

University of Waikato

Lynne Chepulis ( $\square$ lynnec@waikato.ac.nz)

University of Waikato https://orcid.org/0000-0002-9661-4669

Matthew Driller

La Trobe University

Ryan G. Paul

Waikato District Health Board

\section{Short communication}

Keywords: hypoglycaemia, hyperglycaemia, glycaemic control, insulin, carbohydrates

Posted Date: June 15th, 2020

DOI: https://doi.org/10.21203/rs.3.rs-33528/v1

License: (c) (i) This work is licensed under a Creative Commons Attribution 4.0 International License. Read Full License 


\section{Abstract}

In a randomised, counterbalanced, crossover design, eight men with type 1 diabetes (T1D; mean \pm SD age: $27.6 \pm 11.4$ years) reduced insulin (INS) or consumed carbohydrates without the usual insulin bolus (CARBS) over two sessions, held a week apart. Each session included standardised meals, a 45-minute treadmill-walk at $7.24 \mathrm{~km} \cdot \mathrm{h}^{-1}$ and a six-minute walk test (6MWT). Rate of perceived exertion (RPE), blood glucose, blood ketone and blood lactate measures were taken before, during and immediately after the aerobic exercise. The distance covered and the predicted $\mathrm{VO}_{2}$ max were also calculated for the 6MWT. Participants completing the INS intervention spent more time in normoglycaemia $(P<0.01)$ and less time in hyperglycaemia $(P=0.01)$ as compared to the CARBS intervention. Mild hypoglycaemia occurred in two participants during INS and no participants during CARBS. Furthermore, there was no significant difference for blood lactate, ketone, $\mathrm{RPE}$, distance covered and predicted $\mathrm{VO}_{2}$ max between interventions. Six of the eight participants felt that their performance was better during INS, with all six (including the two participants that experienced mild hypoglycaemia) indicating that they would prefer to use this strategy for management of glycaemic during exercise in the future. Based on this pilot study, INS intervention appears to be the best approach for maintaining blood glucose levels in those with T1D during aerobic exercise, though this does need evaluating in other groups, including women, children and those with sub-optimal glycaemic control.

Trial registration: ACTRN12619001397101p. Registered 09 September 2019, http://anzctr.org.au/Trial/Registration/TrialReview.aspx?id=378264

\section{Key Points:}

- Reducing insulin appears to be more effective than eating carbohydrates without the usual insulin bolus for maintaining glycaemic control during aerobic exercise.

- Reduced insulin was the preferred intervention with regard to patient-perceived overall wellbeing, performance and preference for future use.

\section{Introduction:}

Exercise is a cornerstone of management of type 1 diabetes (T1D) as it can aid with glycaemic control, reduce cardiovascular disease and improve quality of life [1-3]. However, many patients with T1D are concerned about undertaking exercise because of the risk of developing hypoglycaemia and how to adjust their insulin or carbohydrate intake to prevent aberrations in their glycaemic control $[3,4]$. Indeed, in a systematic review of 10 studies, the effects of physical exercise and recovery in T1D were shown to be dependent on exercise scheduling, duration and intensity of the exercise, prior carbohydrate consumption, insulin therapy, pre-exercise glucose levels, and cardiovascular fitness [5]. However, with an adequate knowledge of metabolic responses and appropriate diabetes self-management, patients with T1D should be able overcome their fear of exercise-induced dysglycaemia [3, 4]. 
Guidelines recommend that to prevent exercise-induced hypoglycaemia, patients with T1D must either reduce their basal insulin doses or consume carbohydrates without co-administration of insulin prior to commencing exercise [6, 7]. However, whilst research does indicate that both strategies can independently support glycaemic control during exercise $[8,9]$. Knowledge of whether one strategy is better than the other is lacking, and limited peer-reviewed studies have compared both approaches during exercise within the same experimental design [10]. Thus, the aim of the current study was to evaluate the effects of these two approaches on glycaemic control (time in normo-, hypo- and hyper-glycaemia) and performance in a single bout of aerobic exercise. We also measured the effects of the two interventions on predicted $\mathrm{VO}_{2}$ max in a six-minute maximum walk test (6MWT), and on patient-perceived performance.

\section{Methods:}

\section{Participants:}

Men with T1D were recruited directly from the Waikato Regional Diabetes Service (WRDS, Hamilton, New Zealand) between September and November 2019. To be eligible for inclusion, all participants needed to have had T1D for at least two years, be aged 18-60 years, have had a recent $\mathrm{HbA} 1 \mathrm{c}$ (within the last 3 months) of $45-90 \mathrm{mmol} / \mathrm{mol}$; be able to walk/run unaided for 45 minutes at a speed of $7.24 \mathrm{~km} \cdot \mathrm{h}^{-1}$ and be fully competent with diabetes self-management. Participants were excluded if they were taking testosterone therapy, had any comorbidities that would impact on their ability to complete the study (e.g. cardiovascular disease, cerebrovascular disease, severe diabetic retinopathy, nephropathy, neuropathy); were taking medications that altered heart rate (e.g. beta-blockers); or were currently using closed-loop insulin pump therapy. All potential participants were screened at the WRDS Clinic and upon recruitment were required to attend the clinic again 38-48 hours prior to the first test session to have a Flash Glucose monitoring (FGM) device (Abbott Freestyle Libre, Chicago, IL) inserted to allow for continuous glucose monitoring [11]. All participants were able to wear the Freestyle Libre device for 14 days from the date of insertion. The study was successfully registered under the Australia New Zealand Clinical Trial Registry (ACTRN12619001397101p) and was approved by the New Zealand Health and Disability Ethics Committee (ref 19/NTB/175).

\section{Study Design:}

This study was a randomised, counterbalanced, crossover design, in which men with T1D evaluated two different interventions for managing glycaemia whilst undertaking a pre-defined exercise protocol. The two sessions were carried out a week apart at the University of Waikato gymnasium in a controlled, indoor environment with meals (breakfast and lunch) provided.

The night before each session, participants were required to consume a meal that contained at least $1 \mathrm{~g}$ of carbohydrate/kg of body weight [6], and they were asked to eat the same meal before both intervention sessions. Consumption of alcohol, caffeine-based beverages and performing strenuous exercise were 
prohibited during the 12 hours before each aerobic exercise session. Participants then fasted following dinner on the previous day until 9am the following morning.

The two interventions assessed were i) reducing their basal insulin (INS) or ii) consuming carbohydrates without the usual insulin bolus (CARBS)) along with the breakfast meal. Breakfast was provided at 9 am, and consisted of bananas, apple juice and 100\% Isolate protein powder (Musclepharm COMBAT, New Zealand), with quantities individualised to contain $0.66 \mathrm{~g}$ of carbohydrate/ $\mathrm{kg}$ of body weight and $3.375 \mathrm{kcal} / \mathrm{kg}$ of body weight, meeting both the requirements of exercise carbohydrates guidelines [6] and calculated energy expenditure using the Browning Walking Metabolic Prediction equation [12].

During the INS intervention, participants were required to reduce their insulin by $50 \%$ of their normal prandial dose immediately before the breakfast meal as a single bolus as per the consensus guidelines [7]. Those participants in the INS intervention on continuous subcutaneous infusion of insulin (CSIl; insulin pump therapy) started a $50 \%$ temporary basal rate reduction 90 minutes before aerobic exercise, until 60 minutes after completion of the aerobic exercise [7]. The participants in the CARBS intervention consumed their breakfast meal without the administration of any prandial insulin [6]. Throughout the test sessions the participants were not allowed to further adjust their insulin doses or carbohydrates intake. Any adjustments (required or accidental) would trigger the cessation of the session for this participant.

All participants began the aerobic exercise at $10.00 \mathrm{am}$ and were required to walk on a motorised treadmill (Life Fitness, Chicago, IL; USA) at a fixed speed of $7.24 \mathrm{~km} \cdot \mathrm{h}^{-1}$ (4.5 miles per hour) at a $1 \%$ gradient for 45 minutes. Ten minutes following completion of the treadmill walk, participants then performed the 6MWT [13] by walking as quickly as possible for six minutes around the perimeter of an inside arena. This test is considered as a validated measure of predicted VO2 max, and this was calculated as reported previously [14]. For the 6MWT distances were marked off every $3 \mathrm{~m}$, and the total distance walked in the six minutes was recorded. Approximately sixty minutes after completion of the exercise activities, participants had lunch (One Square Meal, OSM: Queenstown, New Zealand), individualised to consist of $1 \mathrm{~g}$ of carbohydrate $/ \mathrm{kg}$ of body weight and administered their normal dose of bolus insulin [7]. Water was allowed ad libitum throughout each session and no additional adjustments (including self-adjustment) of insulin or carbohydrate were allowed until three hours after lunch.

Capillary blood glucose was initially measured before breakfast. Capillary glucose and lactate were measured immediately at the start of the 45-minute aerobic exercise, every 15 minutes during the aerobic exercise, at the end of the 45-minute aerobic exercise, within 1-3 minutes after completion of the 6MWT [15] and before lunch. Blood ketone level measurements were taken at the beginning and the end of the 45-minute aerobic exercise and within 1-3 minutes after completion of the 6MWT. Blood lactate was measured using a lactate analyser (Lactate Pro 2, Arkray, Japan). Glucose levels were monitored directly using the FGM device, and also via capillary sampling (Abbott Optium glucose strips) to minimise any delay between the interstitial and blood glucose levels during physical activity [11]. Self-perceived rate of exertion (RPE) was also assessed every 15 minutes during the aerobic exercise, and immediately following the 6MWT using the Borg's RPE scale [16]. After completion of both sessions', participants were 
asked to self-report which intervention, if either, they preferred and which they were likely to adopt for future exercise.

\section{Statistical Analysis:}

Descriptive statistics and data are expressed as mean \pm standard deviation (SD). Paired T-tests (twotailed) were performed on blood glucose levels, blood ketone, blood lactate, RPE, distance covered and the predicted $\mathrm{VO}_{2}$ max for the two interventions. Thresholds for normal range or normoglycaemia were 3.9-10 mmol/L; mild hyperglycaemia between 10.1-13.9 mmol/L; severe hyperglycaemia $\geq 14 \mathrm{mmol} / \mathrm{L}$; and hypoglycaemia $<3.9 \mathrm{mmol} / \mathrm{L}$. Statistical significance was accepted at a level of $\mathrm{P}<0.05$.

\section{Results:}

Eight men with T1D participated in the study (age: $27.6 \pm 11.4$ years; BMl: $26.6 \pm 2.2 \mathrm{~kg} . \mathrm{m}^{2}$; HbA1c: $55.1 \pm$ $7.4 \mathrm{mmol} / \mathrm{mol}$ ) and all were able to complete both sessions without the need for additional insulin correction. Six participants were on multiple daily injections of insulin (MDI) and two participants on CSII.

Overall, participants completing the INS intervention spent more time in normoglycaemia ( $242 \pm 135$ vs $88 \pm 132$ mins; $P<0.01)$ and less time in severe hyperglycaemia $(41 \pm 95$ vs $154 \pm 125 ; P=0.01)$ compared to the CARBS intervention (Figure 1). The time in mild hyperglycaemia was also halved during the INS intervention (mean $66 \pm 76$ vs $133 \pm 111$ ), though this was not statistically different $(P=0.23$ ). Mild hypoglycaemia occurred in two participants during the INS intervention and no participants during CARBS ( $26 \pm 45$ vs 0 minutes; $P=0.17$ ). Mean blood glucose levels remained significantly higher during CARBS than INS from 15 mins into the aerobic exercise until the duration of the study, despite a comparable blood glucose level just prior to breakfast (Figure 1).

There were no significant differences in blood lactate, ketone levels or rate of perceived exertion (RPE) during the treadmill test at any timepoints between interventions (all $P>0.05$ ). However, participants completing INS intervention were likely able to walk further in the 6MWT (mean \pm SD $777.9 \pm 319.9$ vs $724.6 \pm 302.2, \mathrm{P}=0.07$ ) with no change in predicted $\mathrm{VO}_{2} \max ($ mean $\pm \mathrm{SD} 39.7 \pm 16.2$ vs $38.8 \pm 16.0, \mathrm{P}=$ 0.38) compared to the CARBS intervention.

Self-reported preferences indicated that $75 \%$ of participants felt that their performance was better during INS, with all six (including the two participants that experienced mild hypoglycaemia) indicating that they would use this strategy for management of glycaemic during exercise in the future.

\section{Discussion:}

Only limited studies have evaluated the effects of both reducing insulin and eating carbohydrates without the usual insulin bolus on glycaemic control and performance with aerobic exercise [7]. Here we compare these two recommendations and demonstrate that reducing insulin prior to exercising is the best strategy for maintaining normoglycaemia and likely the better strategy for optimising aerobic performance in 
patients with T1D. Furthermore, reducing insulin by $50 \%$ did not increase the risk of ketoacidosis or lactatemia, and was the preferred strategy for most participants. Others have also shown that reducing insulin is the best strategy for preventing hyperglycaemia $[9,17]$, but that consuming extra carbohydrates is the safest option in preventing hypoglycaemia $[10,18]$. However, as in this study, athletes with diabetes often prefer the risk of mild hypoglycaemia than hyperglycaemia due to the deleterious effects of the latter on exercise performance [19]. Nevertheless, it is clear that patients with T1D need individualised reductions in their insulin around exercise and the priority in the majority of patients will be preventing hypoglycaemia rather than performance [7].

Despite only being a small pilot study, strengths of this study include that participants acted as their own controls in a direct comparison of the two main approaches in T1D, and that unlike other studies, both groups had similar glucose levels at baseline. However, this study did not include women (to minimise the effects of the sex hormones), children or the elderly, and further larger studies are required in both male and female patients across the life span. All participants in this study also had good glycaemic control, so it would be worthwhile comparing these guideline recommendations in patients with suboptimal glycaemic control, because in many of these patients' reductions in insulin or extra carbohydrates may not be required, particularly with short periods of exercise. Lastly, the recommendations will likely differ depending on the different types, intensity and length of exercise.

\section{Declarations:}

\section{Ethics Approval and Consent to Participate:}

This study was approved by the New Zealand Health and Disability Ethics Committee (HDEC) (ref 19/NTB/175) and conforms to the Declaration of Helsinki. Prior to participation, written informed consent was obtained.

\section{Consent for Publication:}

All authors have reviewed the manuscript and approve it for publication. No individual data is presented in this manuscript.

\section{Availability of data and materials:}

The datasets used and/or analysed during the current study are available from the corresponding author on reasonable request.

\section{Competing Interests:}

The authors declare that they have no competing interests 


\section{Funding:}

This study was funded by the Waikato Regional Diabetes Service, Waikato District Health Board, Hamilton, New Zealand.

\section{Author Contributions:}

$\mathrm{RP}, \mathrm{LC}$ and MD designed the study and all authors were involved in the collection of data. VV analysed the data, and LC, RP and VV prepared the manuscript for publication. All authors approve the final manuscript.

\section{Acknowledgements:}

We would like to thank the University of Waikato for providing a student scholarship to support VV during this study.

\section{List Of Abbreviations}

T1D Type 1 Diabetes

6MWT 6 Minute Walk Test

INS Reduced Insulin Intervention

CARBS Additional carbohydrates intervention

CSII Continuous subcutaneous infusion of insulin

$\mathrm{VO}_{2} \max \quad$ Maximal oxygen consumption

RPE Rate of perceived exertion

WRDS Waikato Regional Diabetes Service

HbA1c Glycated haemoglobin

\section{References}

1. Stel G, Francescato MP, Stenner E, Geat M. Prolonged Exercise in Type 1 Diabetes: Performance of a Customizable Algorithm to Estimate the Carbohydrate Supplements to Minimize Glycemic Imbalances. PLoS One. 2015;10(4):e0125220. 
2. WróBel M, Rokicka D, Czuba M, Gołaś A, Pyka $Ł$, Greif M, et al. Aerobic as well as resistance exercises are good for patients with type 1 diabetes. Diabetes Res Clin Pract. 2018;144:93-101.

3. Hasan S, Shaw S, Gelling L, Kerr C, Meads C. Exercise modes and their association with hypoglycemia episodes in adults with type 1 diabetes mellitus: a systematic review. BMJ Open Diabetes Research Care. 2018;6(1):e000578.

4. Codella R, Terruzzi I, Luzi L. Why should people with type 1 diabetes exercise regularly? Acta Diabetol. 2017;54(7):615-30.

5. García-García F, Kumareswaran K, Hovorka R, Hernando M. Quantifying the Acute Changes in Glucose with Exercise in Type 1 Diabetes: A Systematic Review and Meta-Analysis. Sports Med. 2015;45(4):587-99.

6. Samsum Diabetes Research Institute. ExCarbs. Available from https://excarbs.sansum.org/ [accessed May 2020] 2018 [.

7. Gallen IW, Smart CE, Taplin CE, Adolfsson P, Lumb AN, Kowalski A, et al. Exercise management in type 1 diabetes: A consensus statement. The Lancet Diabetes Endocrinology. 2017;5(5):377-90.

8. Adolfsson P, Mattsson S, Jendle JJEjoap. Evaluation of glucose control when a new strategy of increased carbohydrate supply is implemented during prolonged physical exercise in type 1 diabetes. 2015;115(12):2599-607.

9. Zaharieva D, Yavelberg L, Jamnik V, Cinar A, Turksoy K, Riddell MCJDt, et al. The effects of basal insulin suspension at the start of exercise on blood glucose levels during continuous versus circuitbased exercise in individuals with type 1 diabetes on continuous subcutaneous insulin infusion. 2017;19(6):370-8.

10. Grimm J, Ybarra J, Berné C, Muchnick S, Golay A. A new table for prevention of hypoglycaemia during physical activity in type 1 diabetic patients. Diabetes Metabolism. 2004;30(5):465-70.

11. Zaharieva DP, Riddell MC. Henske JJJods, technology. The accuracy of continuous glucose monitoring and flash glucose monitoring during aerobic exercise in type 1 diabetes. 2019;13(1):140.

12. Dubé MC, Prud'homme D, Lemieux S, Lavoie C, Weisnagel SJ. Relation between energy intake and glycemic control in physically active young adults with type 1 diabetes. Journal of Science Medicine in Sport. 2014;17(1):47-50.

13. Burr JF, Bredin SSD, Faktor MD, Warburton DER. The 6-Minute Walk Test as a Predictor of Objectively Measured Aerobic Fitness in Healthy Working-Aged Adults. The Physician Sportsmedicine. $2011 ; 39(2): 133-9$.

14. Ross RM, Murthy JN, Wollak ID, Jackson, ASJBpm. The six minute walk test accurately estimates mean peak oxygen uptake. 2010;10(1):31.

15. Goodwin ML, Harris JE, Hernández A, Gladden LB. Blood lactate measurements and analysis during exercise: A guide for clinicians. Journal of Diabetes Science Technology. 2007;1(4):558-69.

16. Williams N. The Borg Rating of Perceived Exertion (RPE) scale. Occup Med. 2017;67(5):404-5. 
17. Mauvais-Jarvis F, Sobngwi E, Porcher R, Garnier JP, Vexiau P, Duvallet A, et al. Glucose response to intense aerobic exercise in type 1 diabetes: maintenance of near euglycemia despite a drastic decrease in insulin dose. 2003;26(4):1316-7.

18. Riddell MC. Milliken JJDt, therapeutics. Preventing exercise-induced hypoglycemia in type 1 diabetes using real-time continuous glucose monitoring and a new carbohydrate intake algorithm: an observational field study. 2011;13(8):819-25.

19. Shugart C, Jackson J, Fields KBJSh. Diabetes in sports. 2010;2(1):29-38.

\section{Figures}

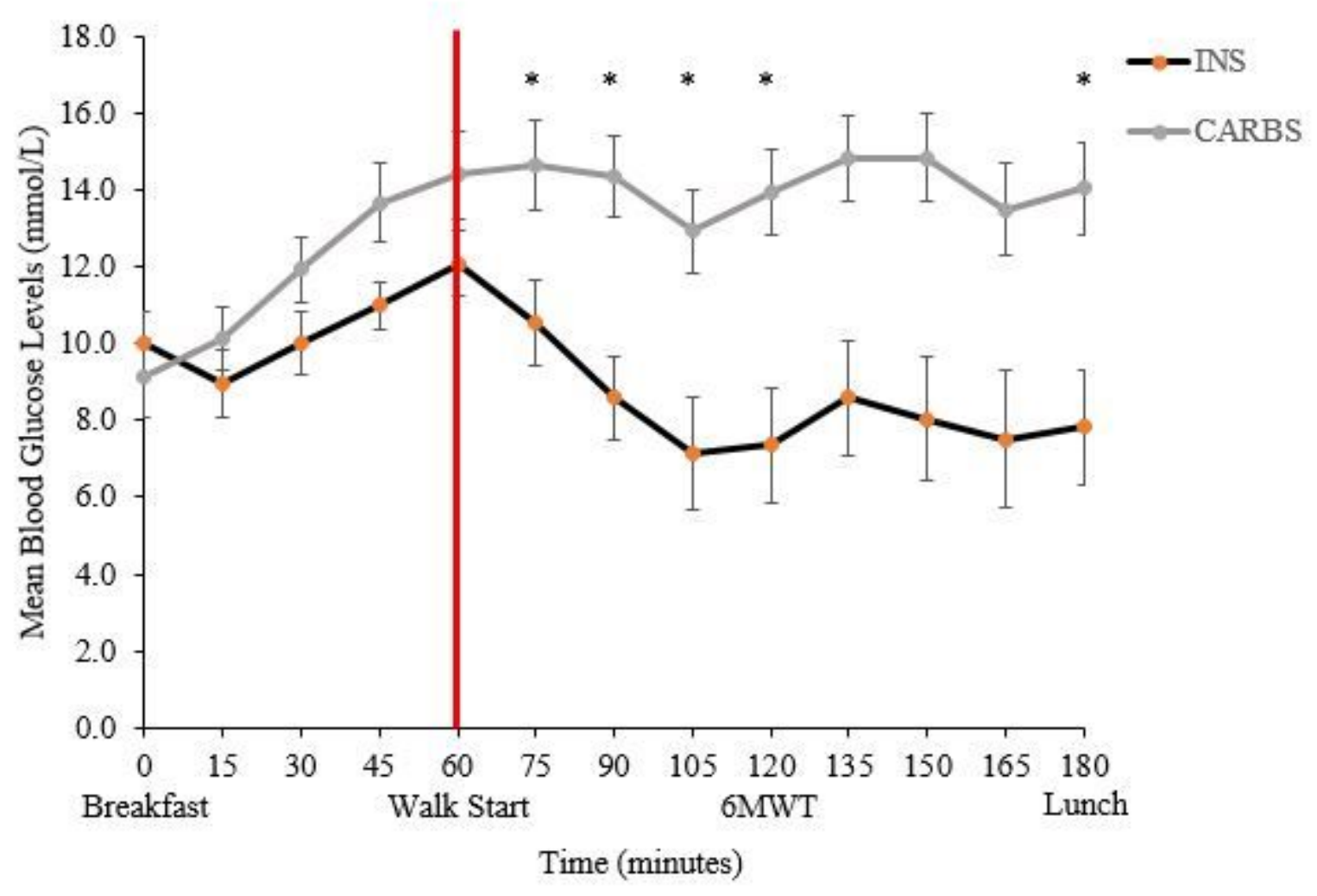

\section{Figure 1}

The effects of reducing bolus insulin with (INS) vs consuming carbs without insulin (CARBS) on blood glucose levels. ${ }^{*} \mathrm{P}<0.05$ between INS and CARBS 